\title{
Development of object recognition in humans
}

\section{Mayu Nishimura*, Suzy Scherf and Marlene Behrmann}

\author{
Address: Department of Psychology, Carnegie Mellon University, Pittsburgh, PA 15213, USA \\ * Corresponding author: Mayu Nishimura (mayu@andrew.cmu.edu) \\ FI000 Biology Reports 2009, I:56 (doi:I0.34I0/BI-56)
}

The electronic version of this article is the complete one and can be found at: http://FI000.com/Reports/Biology/content/I/56

\begin{abstract}
Although the ability to perceive simple shapes emerges in infancy, the ability to recognize individual objects as well as adults do continues to develop through childhood into adolescence. Despite this slow development, recent neuroimaging studies have revealed that an area of the ventral visual cortex that responds selectively to the category of common objects is adult-like by $5-8$ years of age. The challenge for future research will be to identify the specific visual skills involved in object recognition that continue to develop through childhood and adolescence, and the neural mechanisms underlying this protracted development.
\end{abstract}

\section{Introduction and context}

Adults have a remarkable ability to remember thousands of objects in great detail [1]. In addition to having an accurate visual memory of a particular object, adults have a flexible representation of objects that allows recognition of familiar objects under various viewing conditions (for example, lighting, viewpoint, color, retinal size) that change the retinal image. How such accurate and efficient recognition is accomplished is a question that has yet to be resolved [2], but new insights may be gained by examining how this ability develops with age. Consistent with the findings from the immense number of studies on face recognition and perception [3], the few studies examining non-face object perception and recognition reveal continuing improvement with age from young childhood into adulthood.

The antecedents of object recognition are evident in early infancy. For example, newborns preferentially orient towards visual stimuli with a face-like structure [4-6], which may provide an important diet of biased visual experience that supports later face (and perhaps object) recognition $[7,8]$. By 3-4 months of age infants can recognize three-dimensional shapes [9]. They also appear to have some understanding of shape parts when familiarized with a compound object, infants show subsequent recognition of the component two- dimensional shapes that went into forming the compound object [10]. This kind of form perception continues to improve rapidly along with more general visual abilities, such that by 6 years of age grating acuity and contrast sensitivity are adult-like [11], and by 9 years of age children are adult-like in perceiving global form in Glass patterns [12]. However, the perception (for example, classifying objects based on similarity) and recognition (for example, naming a familiar object) of more complex objects (for example, bicycles, teddy bears, abstract three-dimensional shapes) improves with age from young childhood to adolescence $[13,14]$.

In particular, what appears to develop slowly are two important abilities involved in processing objects: the ability to differentiate exemplars within a particular object category (for example, two different cups), and the ability to recognize the same exemplar from multiple viewpoints that change the object's appearance quite dramatically (for example, a teapot seen from above looks quite different from the canonical side view). Describing the mechanisms underlying these abilities also provides the biggest challenge for studies of object recognition in the adult brain [2]. Developmental research with face stimuli has shown that children are particularly poor at processing the spatial relations among facial features [15]. This is arguably a critical 
skill for object recognition more generally because two exemplars of the same category often differ primarily on the spatial relations among the object features, and because knowledge about the structural relations of salient features supports extrapolation to novel or unfamiliar views. Indeed, young children's similarity judgments about objects appear to rely more on the shape of salient features than on the spatial arrangement of those features $[16,17]$, and sensitivity to the spatial arrangement of features continues to improve into adolescence $[18,19]$. However, it is unclear to what extent such age-effects reflect improvements in object perception per se, and/or more general visual abilities associated with form perception such as contour integration $[20,21]$, and/or cognitive limitations in the ability to attend to and/or remember multiple features simultaneously $[22,23]$. Given that many aspects of perception and cognition improve with age, drawing conclusions about the development of object recognition per se is inherently difficult and requires appropriate control stimuli and tasks.

\section{Major recent advances}

In adults, efficient object recognition is supported, in part, through a division of labor, such that different classes of visual input are assigned to different underlying neural systems to mediate the representation of that object type [24]. For example, recent studies using functional magnetic resonance imaging (fMRI) have revealed category-specific activation in adult ventral visual cortex when viewing common objects, faces, buildings, and scrambled image patterns. This foundation of work is ideal for assessing potential developmental changes in the functional topography of ventral visual cortex. In adults, faces consistently activate a lateral portion of the posterior fusiform gyrus, called the fusiform face area [25], a lateral region in inferior occipital cortex called the occipital face area [26], and the superior temporal sulcus [27], whereas non-face objects primarily activate a region of the lateral occiptal cortex (LOC) [28], and images of buildings and scenes activate a region of the parahippocampal gyrus called the parahippocampal place area [29]. Two recent neuroimaging studies have shown that children as young as 5-8 years of age demonstrate adult-like category-selectivity for objects in the LOC $[30,31]$. This finding is in striking contrast to the relatively slow development of the face-selective areas, which are smaller and vary greatly in location in young children and even in adolescents up to 16 years of age compared with adults $[30,31]$. However, it is unclear what early maturation of area LOC represents in terms of recognizing individual objects (as opposed to object categories) or the same object from various viewpoints, because the LOC is defined simply as the area that shows greater bloodoxygen-level-dependent activation when viewing common objects relative to other classes of visual stimuli. Additionally, when observers are unexpectedly given an old-new recognition task to recall objects seen during the fMRI scan, recognition performance of common objects does not correlate with the size of LOC activation in children, adolescents, or adults (there was also no difference in recognition performance across the three age groups), even though recognition of faces correlated with right fusiform face area activation, and recognition of places correlated with left parahippocampal place area activation [30].

In adults, progress has been made to identify the functional properties of the LOC using fMR-adaptation paradigms [32]. Repeated presentations of the same object image suppress LOC activation relative to sequential presentations of different objects. To the extent that this suppression effect is maintained even as changes are made to a particular object (for example, size, position, viewpoint), rather than 'released', the LOC is said to be invariant to such image properties $[28,32]$. Previous studies with adults showed that the LOC is invariant to changes in object size and position, but not viewpoint $[28,32,33]$. Whether such adaptation effects will be observed in children and adolescents remains to be tested. Findings from such studies may reveal limitations in the neural basis of invariant object representations in children, which would be consistent with the behavioral evidence of delayed object perception.

\section{Future directions}

In summary, the behavioral research suggests that the ability to recognize objects continues to develop from childhood into adolescence, and results from developmental fMRI studies reveal that area LOC shows robust category selectivity for common objects in children as young as 5-8 years of age. Nothing is currently known about developmental changes in the neural substrate supporting the formation of integrated invariant representations of individual objects, or faces for that matter. The challenge for future studies then, is to combine finegrained behavioral measurements with neuroimaging techniques so that we can begin to understand how such representations and their neural foundation emerge developmentally.

Currently, there is a clear lack of behavioral research in young children (age 2-6 years) and adolescents (age 12-16 years) with appropriate control tasks to determine their ability to recognize objects despite concomitant immaturities in general visual abilities, attention, and 
memory. Recently, paradigms utilizing adaptation aftereffects have been used successfully with face stimuli [34] and may also be useful in examining the underlying mental representation of common objects. In addition, studies that use naturalistic stimuli, such as videos of objects from multiple views within a scene, may be important for future developmental research.

There is also very little known about the neural mechanisms underlying object recognition in infants and young children. The existing studies have been conducted in the context of understanding memory development and have primarily evaluated infants' and children's abilities to distinguish familiar from unfamiliar stimuli. These studies suggest that brain activity, as measured by event-related potentials, evoked by visual processing of faces and common objects, develops greatly during the first year of life [35]. Infants' eventrelated potentials (ERPs) can also reliably discriminate a highly familiar (for example, infant's mother's face or infant's favorite toy) versus a novel exemplar (for example, an unfamiliar female face or toy) by 18 months of age [36]. Therefore, electroencephalography may be a particularly useful method for examining the neural correlates of object recognition in infants and young children. Future studies should investigate how well infants discriminate and recognize entirely novel exemplars within an object category.

Another potentially exciting avenue for future research is to examine dorsal stream contributions to the development of object recognition. There is some suggestion that action perception may aid in young children's object recognition [37]. Consistent with the notion that action may influence perception, fMR-adaptation has been used to reveal the existence of object representations in the dorsal stream of adults [33]. If areas in the dorsal stream that are relevant for object recognition mature earlier than the ventral stream, as has been suggested [38], then the interactions between the dorsal and ventral streams may play a large role in bootstrapping the acquisition of invariant object representations.

The key for future research will be studies that correlate neural activity, as measured by fMRI, ERP, or even magnetoencephalography, with behavioral performance in the same infants/children to elucidate the development of visual mechanisms subserving object recognition. Findings from these investigations will provide a foundation of knowledge about one of the most essential questions in the cognitive and neural sciences, namely, how accurate and efficient object recognition is accomplished. Such work will also provide a benchmark for assessing deviations in many neurodevelopmental disorders that affect visuoperceptual processing, like autism and Williams syndrome. In addition, identifying the neural regions supporting behavioral development of object recognition will direct targeted investigations of the neural code underlying adult object recognition, which could, in turn, provide important insights for implementing machine vision. Critical to the goal of developing a comprehensive theory of the emergence and instantiation of sophisticated object recognition abilities is to ensure consistency and transparency of methods and stimuli so that results may be comparable across different samples and age ranges.

\section{Abbreviations}

ERP, event-related potential; fMRI, functional magnetic resonance imaging; LOC, lateral occipital cortex.

\section{Competing interests}

The authors declare that they have no competing interests.

\section{Acknowledgments}

This work was funded by a National Institute of Mental Health grant (MH54246) to MB.

\section{References}

I. Brady TF, Konkle T, Alvarez GA, Oliva A: Visual long-term memory has massive storage capacity for object details. Proc Natl Acad Sci U S A 2008, I05:|4324-9.

2. Peissig JJ, Tarr MJ: Visual object recognition: Do we know more now than we did 20 years ago? Annu Rev Psychol 2007, 58:75-96.

3. Mondloch CJ, Geldart S, Maurer D, Le Grand R: Developmental changes in face processing skills. J Exp Child Psychol 2003, 86:67-84.

4. Johnson $\mathrm{MH}$, Dziurawiec S, Ellis H, Morton J: Newborns' preferential tracking of face-like stimuli and its subsequent decline. Cognition 1991, 40:I-19.

5. Cassia VM, Turati C, Simion F: Can a nonspecific bias toward topheavy patterns explain newborns' face preference? Psychol Sci 2004, I 5:379-83

6. Farroni T, Johnson MH, Menon E, Zulian L, Faraguna D, Csibra G: Newborns' preference for face-relevant stimuli: Effects of contrast polarity. Proc Natl Acad Sci U S A 2005, I02: I7245-50.

7. Le Grand R, Mondloch CM, Maurer D, Brent HP: Early visual experience and face processing. Nature 200I, 4I 0:890.

8. Le Grand R, Mondloch CM, Maurer D, Brent HP: Impairment in holistic face processing following early visual deprivation. Psychol Sci 2004, I 5:762-8.

9. Kraebel KS, West RN, Gerhardstein P: The influence of training views on infants' long-term memory for simple 3D shapes. Dev Psychobiol 2007, 49:406-20.

10. Haaf RA, Fulkerson AL, Jablonski BJ, Huppa JM, Schull SS, PescaraKovach L: Object recognition and attention to object components by preschool children and 4-month-old infants. J Exp Child Psychol 2003, 86:108-23.

II. Ellemberg D, Lewis TL, Liu CH, Maurer D: Development of spatial and temporal vision during childhood. Vision Res 1999, 39:2325-33.

12. Lewis TL, Ellemberg D, Maurer D, Dirks M, Wilkinson F, Wilson HR: A window on the normal development of sensitivity to global form in Glass patterns. Perception 2004, 33:409- I8. 
13. Bova SM, Fazzi E, Giovenzana A, Montomoli C, Signorini SG, Zoppello M, Lanzi G: The development of visual object recognition in school-age children. Dev Neuropsychol 2007, 31:79-102.

14. Juttner M, Muller A, Rentschler I: A developmental dissociation of view-dependent and view-invariant object recognition in adolescence. Behav Brain Res 2006, I75:420-4.

15. Mondloch CJ, Le Grand R, Maurer D: Configural face processing develops more slowly than featural face processing. Perception 2002, 31 :553-66.

16. Mash C: Multidimensional shape similarity in the development of visual object classification. J Exp Child Psychol 2006, 95:128-52.

17. Thompson LA, Markson L: Developmental changes in the effect of dimensional salience on the discriminability of object relations. J Exp Child Psychol 1998, 70: I-25.

18. Scherf KS, Behrmann M, Kimchi R, Luna B: Emergence of global shape processing continues through adolescence. Child Dev 2009, 80:162-77.

19. Rentschler I, Juttner M, Osman E, Muller A, Caelli T: Development of configural 3D object recognition. Behav Brain Res 2004, I49: I07-II.

20. Kovacs I, Kozma P, Feher A, Benedek G: Late maturation of visual spatial integration in humans. Proc Natl Acad Sci U S A 1999, 96:12204-9.

21. Cory E, Atkinson J, Braddick O, Wattam-Bell J, Guzzetta A, Cioni G: Dorsal and ventral stream sensitivity in normal development and hemiplegia. Neuroreport 2002, 13:843-7.

22. Pereira AF, Smith LB: Developmental changes in visual object recognition between 18 and 24 months of age. Dev Sci 2008, 1 2:67-80.

23. Uttal DH, Gentner D, Liu LL, Lewis AR: Developmental change in children's understanding of the similarity between photographs and their referents. Dev Sci 2008, I I:I56-70.

24. Downing PE, Chan AW, Peelen MV, Dodds CM, Kanwisher N: Domain specificity in visual cortex. Cerebral Cortex 2006, 16:|453-6|.

25. Kanwisher N, McDermott J, Chun MM: The fusiform face area: A module in human extrastriate cortex specialized for face perception. J Neurosci 1997, I7:4302-II.
26. Gauthier I, Tarr MJ, Moylan I, Skudlarski P, Gore JC, Anderson AW: The fusiform 'face area' is part of a network that processes faces at the individual level. J Cogn Neurosci 2000, 12:495-504.

27. Hoffman EA, Haxby JV: Distinct representations of eye gaze and identity in the distributed human neural system for face perception. Nat Neurosci 2000, 3:80-4.

28. Grill-Spector K, Kushnir T, Edelman S, Avidan G, Itzchak Y, Malach R: Differential processing of objects under various viewing conditions in the human lateral occipital complex. Neuron 1999, 24:187-203.

29. Epstein R, Kanwisher N: A cortical representation of the local visual environment. Nature 1998, 392:598-60I.

30. Golarai G, Ghahremani DG, Whitfield-Gabrieli S, Reiss A, Eberhardt JL, Gabrieli JDE, Grill-Spector K: Differential development of high-level visual cortex correlates with categoryspecific recognition memory. Nat Neurosci 2007, 10:5I2-22.

31. Scherf KS, Behrmann M, Humphreys K, Luna B: Visual categoryselectivity for faces, places, and objects emerges along different developmental trajectories. Dev Sci 2007, I0:FI5-F30.

32. Grill-Spector K, Malach R: fMR-adaptation: a tool for studying the functional properties of human cortical neurons. Acta Psychol 2001, 107:293-321.

33. Konen CS, Kastner S: Two hierarchically organized neural systems for object information in human visual cortex. Nat Neurosci 2008, I I:224-31.

34. Nishimura M, Maurer D, Jeffery L, Pellicano E, Rhodes G: Fitting the child's mine to the world: Adaptive norm-based coding of facial identity in 8-year-olds. Dev Sci 2008, I I:620-7.

35. Webb SJ, Long JD, Nelson CA: A longitudinal investigation of visual event-related potentials in the first year of life. Dev Sci 2005, 8:605-16.

36. Carver LJ, Meltzoff AN, Dawson G: Event-related potential (ERP) indices of infants' recognition of familiar and unfamiliar objects in two and three dimensions. Dev Sci 2006, 9:5I-62.

37. Mounoud P, Duscherer K, Moy G, Perraudin S: The influence of action perception on object recognition: $A$ developmental study. Dev Sci 2007, 10:836-52.

38. Kovacs I: Human development of perceptual organization. Vision Res 2000, 40:1301-10. 